BRAZILIAN JOURNAL

$\mathrm{OF}$

RADIATION SCIENCES

03-1A (2015) 01-16

\title{
Aplicabilidade da Lei do Inverso do Quadrado da distância em radiologia convencional e mamografia
}

\author{
G. Hoff ${ }^{\mathrm{a}}$, N.W. Lima ${ }^{\mathrm{b}}$ \\ ${ }^{a}$ Bolsista da CAPES - Projeto No. 161/2012. \\ ${ }^{b}$ Faculdade de Física, PUCRS 90619-900, CEP,Porto Alegre, RS, Brasil \\ lima.nathan@gmail.com
}

\begin{abstract}
The Inverse Square Law (ISL) is a mathematical rule used to adjust the KERMA and exposure to different distances of focal spot having as reference a determined point in space. Taking into account the limitations of this rule and its application, we have as main objective to verify the applicability of ISL to determine exposure on radiodiagnostic area (maximum tensions between $30 \mathrm{kVp}$ and $150 \mathrm{kVp}$ ). Experimental data was collected, deterministic calculation and simulation using Monte Carlo Method (Geant4 toolkit) were applied to conventional radiology and mammography. The experimental data was collected using a calibrated ionizing chamber TNT 12000 from Fluke. The conventional X-ray equipment used was a Multix Top of Siemens, with Tungsten track and total filtration equivalent to $2.5 \mathrm{~mm}$ of aluminum; and the mammographic equipment was a Mammomat Inspiration from Siemens, presenting the track-add filtration combinations of Molybdenum-Molybdenum, Molybdenum-Rhodium, Tungsten-Rhodium. Both equipments have the Quality Control testes in agreement to Brazilian regulations. Based on the results it is possible conclude that the ISL presents lower performance in correct measurements on mammography spectra, i.e. the associated error (differences) achieves a value up to $77.8 \%$ and it can cause significant impact on both areas depending on the spectra energy and distance to correct.
\end{abstract}

Keywords: radiology, mammography, dosimetry 


\section{INTRODUÇÃO}

$\mathrm{Na}$ área de proteção radiológica aplicada ao uso das radiações ionizantes na radiologia diagnóstica, tem-se como base usar dados de KERMA ou de exposição em uma determinada posição para estimar a dose em órgãos internos de sujeitos submetidos a exames de radiodiagnóstico, conforme mencionado por Kramer et al (2004) e Zankl et al (2002). Para tanto, utilizam-se fatores de conversão que transformam a exposição na entrada da pele ou KERMA coletados, em um ponto específico, como base de cálculo para estimar a dose absorvida por um órgão ou tecido. Esse modelo, por si só, já apresenta limitações de contorno e precisa, para correta aplicação do fator de conversão, que se conheçam as condições de determinação do fator de conversão (geometria, consideração ou não de retroespalhamento na medida experimental de entrada de dados) conforme descrito por Dance (2005), EUREF (1996) e Hoff (2000).

$\mathrm{Na}$ área da mamografia, por exemplo, a grandeza dose glandular $\left(\mathrm{D}_{\mathrm{g}}\right)$ pode ser definida como a dose absorvida pelo tecido glandular da mama (Wu et al, 1994; Gingold, 1995; Ng, 2000), considerado radiossensível, podendo essa ser determinada através da medida da exposição na entrada da pele $\left(\mathrm{X}_{\mathrm{ESE}}\right)$ e os valores tabelados de dose glandular normalizadas $\left(\mathrm{D}_{\mathrm{gN}}\right)$, conforme mostra a Equação 1.

$$
\overline{D_{g}}=D_{g N} X_{E S E}
$$

No referido modelo, a unidade de medida da grandeza dosimétrica $\mathrm{D}_{\mathrm{g}}$ é joule/quilograma $(\mathrm{J} / \mathrm{kg}$, que recebe a denominação Gray $(\mathrm{Gy}))$. Entretanto, cuidados devem ser tomados na determinação da $\mathrm{D}_{\mathrm{gN}}$ no que se refere às estimativas da tensão de pico do espectro gerado pelo tubo de raios X, a Camada Semi-Redutora (CSR) e a composição da mama, pois estes são fatores determinantes na função de normalização da $\mathrm{D}_{\mathrm{g}}$. Neste caso a $\mathrm{D}_{\mathrm{gN}}$, para um espectro policromático calculado através dos valores de $\mathrm{D}_{\mathrm{gN}}$ para cada energia componente desse espectro deve ser adequadamente ponderada pela função representativa do espectro incidente, conforme a Equação 2 define. 


$$
D_{g N}=\sum_{i} \psi\left(E_{i}\right) D_{g N}\left(E_{i}\right)
$$

Onde $\psi\left(E_{i}\right)$ é a função que define o espectro de raios $\mathrm{X}$ incidente e $\mathrm{D}_{\mathrm{gN}}\left(\mathrm{E}_{\mathrm{i}}\right)$ representa o valor tabelado de $\mathrm{D}_{\mathrm{gN}}$ para um feixe monoenergético $(\mathrm{Ng}, 2000)$. A unidade dessa grandeza é gray/cloulomb/quilograma $[\mathrm{Gy} /(\mathrm{C} / \mathrm{kg})]$.

De acordo com Ng (2000), Wu (1991) e Aus (1999), os valores para $\mathrm{X}_{\mathrm{ESE}}$ devem ser medidos, e uma análise criteriosa dos fatores de erros associados deve ser realizada. Fatores geométricos da câmara de ionização no campo útil de radiação devem ser considerados, pois podem resultar em variação da ordem de $10 \%$ dos valores de exposição (Aus, 1999). A exposição na entrada da pele é associada a cada exposição free in air seguindo as recomendações da Associação Americana de Físicos em Medicina (American Association of Physicists in Medicine, AAPM) (AAPM 1985, AAPM 1989, AAPM 1990).

Pelo modelo preconizado pelo Comitê Europeu, a $D_{g}$, em Gy, pode ser estimada através de cálculos numéricos, como derivada do KERMA incidente no ar $\left(\mathrm{K}_{\mathrm{ai}}\right)$ combinado com o coeficiente de conversão $\left(\mathrm{c}_{\mathrm{g}}\right)$ obtido através dos cálculos de transporte de radiação em modelos voxel de mama, conforme ilustra a relação apresentada na equação a Equação 3.

$$
\overline{D_{g}}=c_{g} K_{a i}
$$

Os cálculos em códigos de transporte de radiação de diversos autores diferem quanto aos modelos de interação dos fótons com a matéria, os espectros, a composição e a espessura da camada superficial (que representa pele e tecido adiposo subcutâneo), a presença do compressor e a distância foco-filme. Além disso, estas simulações não foram realizadas com as composições padrões de materiais recomendadas pela ICRU (1989). Com referência às diferenças entre as 
simulações, valores para $c_{\mathrm{g}}$ variando na ordem de $20 \%$ a $30 \%$ podem ser verificados entre os resultados publicados por diferentes autores, conforme descrito por Hoff (2005).

$\mathrm{Na}$ área da radiologia convencional, um modelo semelhante aos apresentados para mamografia (Equação 4) podem ser aplicados, mas com fatores de conversão diferentes que consideram o corpo todo conforme Kramer et al (2004), Zankl et al (2002) e Takahashi (2011). As considerações de limitações dos modelos supracitados aplicam-se neste modelo também.

$$
D_{\text {orgão }}=c K_{a i}
$$

Com base nos modelos apresentados acima, outros fatores podem ser considerados como a exatidão para a determinação da dose absorvida ou efetiva (dependendo do modelo), a geometria do modelo antropomórfico simulado e sua similaridade com o sujeito de pesquisa e a exatidão da medida de entrada de dados (exposição ou KERMA). Neste trabalho tem-se como objetivo verificar as aproximações consideradas na utilização da Lei do Inverso do Quadrado da Distância (LIQD) em corrigir dados de exposição e KERMA que serão utilizados na estimativa de dose e verificar o impacto dessas considerações na estimativa de dose absorvidas e efetivas.

A LIQD é uma lei matemática que pode ser aplicada a diferentes áreas do conhecimento e se reporta ao comportamento da medida da grandeza, proporcional a emissão de um ente emitido isotropicamente por uma fonte pontual, medida (ou estimada) a distâncias diferentes da fonte emissores do ente. Por definição, essa lei se aplica, sem restrições, a casos de fontes pontuais emitindo isotropicamente no vácuo, pois não são considerados os possíveis espalhamentos do ente emitido no material entre a fonte e o ponto de detecção/contagem. Nessas condições, por causa da geometria do sistema, determina-se que a contagem de entes emitidos (Y), nessas condições, serão proporcionais ao inverso da quadrado da distância (d) do ponto de interesse da fonte emissora (Equação 5)

$$
Y \propto \frac{1}{d^{2}}
$$


A crítica apresentada nesse trabalho busca trazer os limites de aplicabilidade da LIQD em casos reais de aproximação utilizados na medida de exposição em radiologia convencional, onde, mesmo que a fonte real possa ser considerada pontual (em um aproximação matemática), ainda tem-se que avaliar a influência da não homogeneidade de emissão da fonte na área útil (participação do efeito anódico), da atenuação da filtração inerente a adicional utilizadas nos equipamentos de radiologia convencional (na geração de espalhamentos e atenuação real do feixe primário) e a participação da atenuação e do espalhamento do ar na medida das grandezas dosimétricas de base utilizadas (exposição ou KERMA). Essa é uma análise inicial que verifica, de forma geral, a aplicabilidade da LIQD e sua influência nas estimativas de dose absorvida e efetiva por diferentes modelos.

Com base nos modelos acima apresentados que mostram uma relação direta entre a dose absorvida ou efetiva de interesse radiológico, o KERMA ou exposição medidos servem de entrada de dados e se relacionam diretamente com estas grandezas dosimétricas. Assim sendo, erros sistemáticos ou tendências de dados referentes a aplicação de um modelo aproximado devem ser evitados e/ou corrigidos sempre que possível, pois impactarão diretamente no resultado da dose estimada por um método matemático.

\section{MATERIAIS E MÉTODOS}

O trabalho contou com uma avaliação experimental, uma avaliação de modelo simplificado determinístico e a realização de cálculos utilizando o método de Monte Carlo. Para realizar as medidas experimentais de exposição, foi utilizada uma câmara de ionização kit TNT 12000 da Fluke calibrada. O aparelho de raios X convencional utilizado foi um Multix Top da Siemens e o aparelho de mamografia avaliado foi um Mammomat Inspiration da Siemens, ambos equipamentos apresentaram todos os testes de controle da qualidade dentro dos limites estabelecidos pelo Ministério da Saúde (ANVISA, 1998). Quando o estudo foi aplicado à radiologia convencional, as medidas foram realizadas com a distância variável entre o ponto focal e o detector entre $40 \mathrm{~cm}$ e $100 \mathrm{~cm}$, em passos fixos de $10 \mathrm{~cm}$, considerando trilha de Tungstênio, filtração total equivalente a $2,5 \mathrm{~mm}$ de $\mathrm{Al}$ e tensões de pico de $66 \mathrm{kVp}$ e $81 \mathrm{kVp}$. 
Quando o objeto de estudo foi aplicado à mamografia, as medidas foram realizadas considerando distância variável entre o ponto focal e o detector de $26 \mathrm{~cm}, 40 \mathrm{~cm}, 50 \mathrm{~cm}$ e $60 \mathrm{~cm}$; com trilha de Molibdênio ou de Tungstênio, considerando filtração inerente equivalente a $0,8 \mathrm{~mm}$ de Berílio e as possíveis combinações trilha-filtro: Molibdênio-Molibdênio $(25 \mu \mathrm{m})$, Molibdênio-Ródio $(30 \mu \mathrm{m})$, Tungstênio-Ródio $(50 \mu \mathrm{m})$ e tensões de pico de $25 \mathrm{Vp}, 30 \mathrm{kVp}$ e $35 \mathrm{kVp}$

Foram utilizados os espectros do Catalogue of Diagnostic X-ray Spectra and other DataReport 78 do Institute of Physics and Engineering in Medicine organizado por Cranley K. et al (1997) para realização das simulações e cálculos determinísticos. Os espectros utilizados têm as mesmas características dos espectros utilizados no arranjo experimental.

O fator de correção foi analisado com base em um fator normalizador fazendo com que as grandezas fossem consideradas em função da probabilidade por fóton emitido pela fonte, a fim de que o número total de fótons emitidos pela fonte, para cada condição de contorno estudada, não pudesse interferir significativamente no resultado. Isso está relacionada com a dificuldade de definir o número específico de fótons gerados por $\mathrm{mAs} \mathrm{cm}^{2}$ (que está relacionado com a eficiência de geração de radiação $X$ de cada tubo). Assim sendo, para minimizar possíveis erros relacionados a esse fator, a análise de comportamento da curva de exposição foi normalizada para a exposição de referência, tanto na simulação quanto no cálculo determinístico e nos dados experimentais. Desta forma, as alterações verificadas (quando os cálculos foram comparados aos valores experimentais) limitam-se às condições dos modelos aplicados e não fatores externos aos mesmos. A Equação 6 ilustra a consideração de normalização utilizada para todos os casos estudados, onde o $X_{\text {ponto-de-interesse }}$ e $X_{\text {ponto-de-referência }}$ são exposições em pontos no espaço a definir durante a análise.

$$
X_{\text {norm }}=\frac{X_{\text {pontede-interesse }}}{X_{\text {pontede-referêncic }}}
$$

A ferramenta computacional Geant4, descrita por Agostinelli et al (2003) e Allison et al (2006), em sua versão 9.06.p01, foi usada para aplicar Método de Monte Carlo à proposta deste 
trabalho. Uma geometria similar à geometria experimental foi definida no mundo virtual. Isto está representado na Figura 1.

Figura 1. Imagem da geometria de simulação em vista lateral gerada pelo visualizador HepRep, onde o retângulo branco representa a câmara de ionização, o retângulo vermelho representa a filtração adicional do equipamento de raios $\mathrm{X}$ e o retângulo verde representa a filtração inerente do tubo de raios X. As linhas verdes e pontos vermelhos representam fótons se propagando e elétrons secundários gerados durante a simulação.

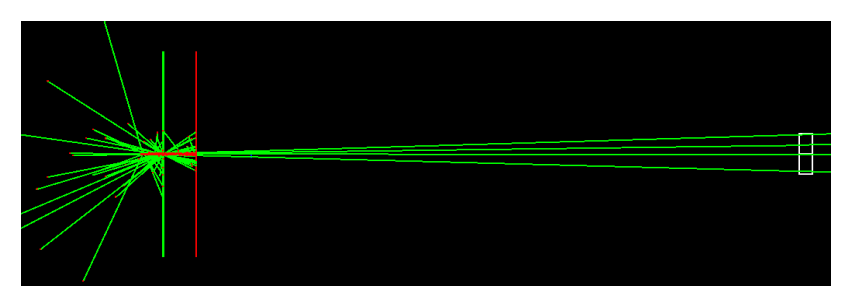

Para descrever o transporte de radiação pela matéria, foi selecionada a biblioteca de secção choque e modelos de baixa energia "Low Energy Livermore Model”. Foram definidos valores de corte de $0,1 \mathrm{~mm}$ para todas as partículas que atingem o volume sensível da câmara de ionização e $0,1 \mathrm{~mm}$ para fótons e $1,0 \mathrm{~m}$ para elétrons para o resto do mundo. $\mathrm{O}$ volume do detector foi definido considerando uma câmara de ionização com 15 cc de volume sensível. A fonte foi definida como pontual irradiando fótons homogeneamente numa área circular de raio $2,08 \mathrm{~cm}$ na entrada da superfície da câmara de ionização. Foram usadas $10^{8}$ histórias para cada run para chegar nos resultados com a estatística adequada.

Foi simulada a energia absorvida pelo volume de interesse e a estimativa da exposição foi realizada através da aplicação da Equação 7, descrita por Attix (1986), que relaciona a exposição (X) com a energia total absorvida $\left(\mathrm{E}_{\mathrm{ab}}\right.$ ), que foi simulada, com o $\mathrm{W}_{\mathrm{par}}=33,94 \mathrm{~J} / \mathrm{C}$ (ATTIX, 1986), representando a energia média gasta pelo gás (no caso ar) para gerar um par elétron-íon positivo. 


$$
X=\frac{E_{a b}}{m_{a r} W_{p a r}}
$$

A Equação 7 foi aplicada para possibilitar a comparação dos resultados experimental e simulados por Método de Monte Carlo e calculados pelo método determinístico. O valor de referência medido foi corrigido para uma nova posição através do fator de correção determinado pela aplicação direta da LIQD, mostrado na Equação 8.

$$
c_{\text {LIQD }}=\frac{\frac{X_{0}}{d_{\text {referência }}^{2}}}{\frac{X_{0}}{d_{\text {interesse }}^{2}}}=\frac{d_{\text {referência }}^{2}}{d_{\text {interesse }}^{2}}
$$

Nas variações ponto a ponto dos dados experimentais foram consideradas as incertezas tipo A e B, sendo utilizado o erro combinado para verificação todo erro experimental que representa a medida. A técnica de Monte Carlo reporta apenas a incerteza proveniente do método de cálculo e distribuição dos dados, não sendo considerados as incertezas epistemológicas inerentes a esses métodos. Já as variações associadas aos dados onde o fator de correção LIQD foi aplicado, o erro percentual da medida de referência como padrão. Entretanto cada ponto normalizado, utilizado na comparação de dados, é proveniente de uma razão entre quantidades medidas e simuladas, sendo assim, utilizou-se a teoria de propagação de erros para definir a variância do dado normalizado.

Para verificação da significância estatística da comparação entre as curvas experimentais e a curva gerada por dados utilizando técnica de Monte Carlo e as curvas experimentais e as curvas corrigidas pela LIQD, utilizou-se o teste de qui-quadrado $\left(\chi^{2}\right)$, incluindo a correção de Yates sempre que o grupo de dados for inferior a 6 dados. Nessa análise, além da comparação utilizar as diferenças entre os dados experimental (esperada) e a curva gerada por métodos de cálculo (observada), observa-se também os erros experimentais e os erros associados ao método de cálculo utilizado. A avaliação de similaridade também foi realizada pelo teste ANOVA.

\section{RESULTADOS E DISCUSSÕES}


Os gráficos apresentados nas Figuras 2 e 3 mostram o comportamento dos dados experimentais normalizados, a simulação computacional (com resultados normalizados) e o fator de correção aplicando-se a LIQD para mamografia considerando como pontos de referência comparativa as distâncias de $26 \mathrm{~cm}$ (Figura 2) e $60 \mathrm{~cm}$ (Figura 3). Em ambos os casos percebe-se que a LIQD apresenta correções significativamente diferentes das baseadas em dados experimentais ou das apresentadas pelas simulações computacionais. Já as simulações computacionais apresentaram dados muito semelhantes aos dados experimentais e, consequentemente, curvas de tendências similares.

Observe que nos gráficos apresentados nas Figuras de 2 a 4 os dados experimentais são apresentados como média dos dados normalizados. Ou seja, os dados de cada ponto representam a média e desvio padrão de cada situação de contorno coletada para aquele ponto (mamografia e radiologia convencional). Entretanto, para que se possa fazer esta consideração, faz-se necessário mostrar que as curvas podem ser representadas desta forma, não sendo elas significativamente diferentes entre si. Para tanto, utilizou-se o teste ANOVA, fator único, para múltiplos exemplos. Para a análise de similaridade das curvas em mamografia, pelo teste ANOVA, as somas dos dados de cada curva variam entre 2,058 e 2,103; as médias variaram entre 0,514 e 0,526; e as variâncias calculadas estão entre 0,110 e 0,115 . Isso, inicialmente, evidencia a similaridade entre as curvas, mas os índice ANOVA Z de 0,000526 (para Z crítico de 3,2558) com valor-p maior que 0,99999 mostram que existe uma concordância com significância maior que 99,99\% de que as curvas normalizadas são iguais. Já as curvas aplicadas à radiologia convencional, pelo teste ANOVA, mostraram somas de dados entre 4,688 e 4,988; as médias variaram entre 0,781 e 0,831; e as variâncias calculadas estão entre 0,029 e 0,892. Isso, inicialmente, evidencia a similaridade entre as curvas, contudo a alteração significativa entre as variâncias mostram o comportamento mais acentuado decrescente para a curve de menor aceleração de pico. Entretanto os índice ANOVA Z de 0,0162 (para Z crítico de 10,044) com valor-p de 0,9012 ainda mostram que existe uma concordância com significância de 90,12\% entre as curvas normalizadas. É importante salientar que a concordância entre as curvas experimentais na área da mamografia é maior de que a concordância entre as curvas normalizadas na área de radiologia convencional. Isso pode ter ocorrido pelas seguintes razões: maiores variações entre as energias 
e as maiores distâncias entre os pontos de medida utilizados área da radiologia convencional. Considerando os resultados apresentados pode-se afirmar que as curvas experimentais para os dados normalizados podem ser consideradas equivalentes, quando avaliadas as curvas de cada aplicação, sendo a representação de média de cada ponto adequada dada a significância da concordância calculada.

Neste momento falta verificar a análise do grau de concordância entre os dados experimentais e os dados calculados com o fator de correção considerando a LIQD e os dados calculados utilizando o Método de Monte Carlo. Como todas as curvas representa um comportamento não linear decrescente (dando a impressão de decrescimento exponencial) ela tem uma certa similaridade entre si. A questão nessa análise é verificar o grau de concordância de cada método de cálculo, incluindo considerações que levem em conta as variações de cada dado normalizado. Assim sendo, foi adicionada a avaliação com teste $\chi^{2}$. Na área da mamografia, verificou-se através do teste ANOVA, com nível de significância 0,01, que a técnica de Monte Carlo teve melhor concordância os dados experimentais com $\mathrm{F}=0,00085$ (para $\mathrm{F}$ crítico 13,745) e confiabilidade de $97,73 \%$, considerando os resultados apresentados pela LIQD com $\mathrm{F}=0,04117$ (para F crítico de 13,745) mas com confiabilidade de 84,59\%. Este teste verifica dados levando em conta somente os dados médios, sem considerar as variações de cada ponto. Assim sendo, aplicou-se o teste $\chi^{2}$ que mostrou também concordância significativa entre os dados gerados pelo método de Monte Carlo com valor-p 0,2391 sendo que o valor-p calculado para LIQD foi inferior a 0,001. A análise da área da radiologia convencional, verificou-se através do teste ANOVA, com nível de significância 0,01, que a técnica de Monte Carlo teve melhor concordância os dados experimentais com $\mathrm{F}=0,0126$ (para $\mathrm{F}$ crítico 10,044) e confiabilidade de $92,12 \%$, considerando os resultados apresentados pela LIQD com F=3,367 (para F crítico de 10,044) mas com confiabilidade de 8,67\%. Este teste verifica dados levando em conta somente os dados médios, sem considerar as variações de cada ponto. Assim sendo, aplicou-se o teste $\chi^{2}$ que mostrou também concordância significativa entre os dados gerados pelo método de Monte Carlo com valor-p 0,064 sendo que o valor-p calculado para LIQD foi inferior a 0,001. Novamente a concordância com os dados de mamografia foi superior (com maior significância estatística) do que a concordância com os dados de radiologia convencional. Isso pode ser resultado, além das variáveis citadas anteriormente, a maior flutuação em torno do valor médio 
experimental, ocasionado pela maior variação apresentada pelas curvas da radiologia convencional.

Figura 2. Gráfico que ilustra o comportamento das curvas das medidas de exposição normalizadas experimental, simulada no Geant4 e com aplicação do fator de correção do LIQD para as energias utilizadas em mamografia considerando a medida/simulação a $26 \mathrm{~cm}$ como referência.

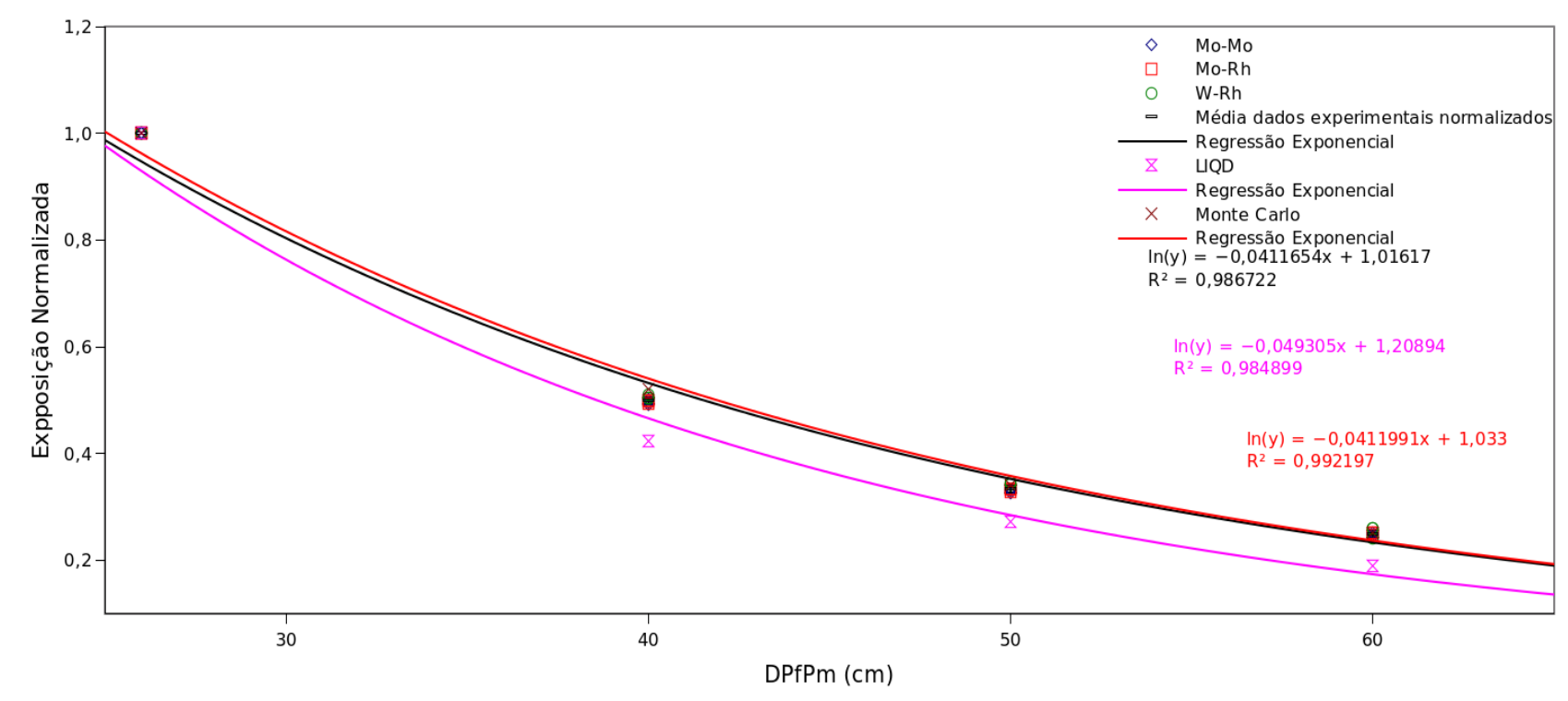

Figura 3. Gráfico que ilustra o comportamento das curvas das medidas de exposição normalizadas experimental, simulada no Geant4 e com aplicação do fator de correção do LIQD para as energias utilizadas em mamografia considerando a medida/simulação a $60 \mathrm{~cm}$ como referência. 


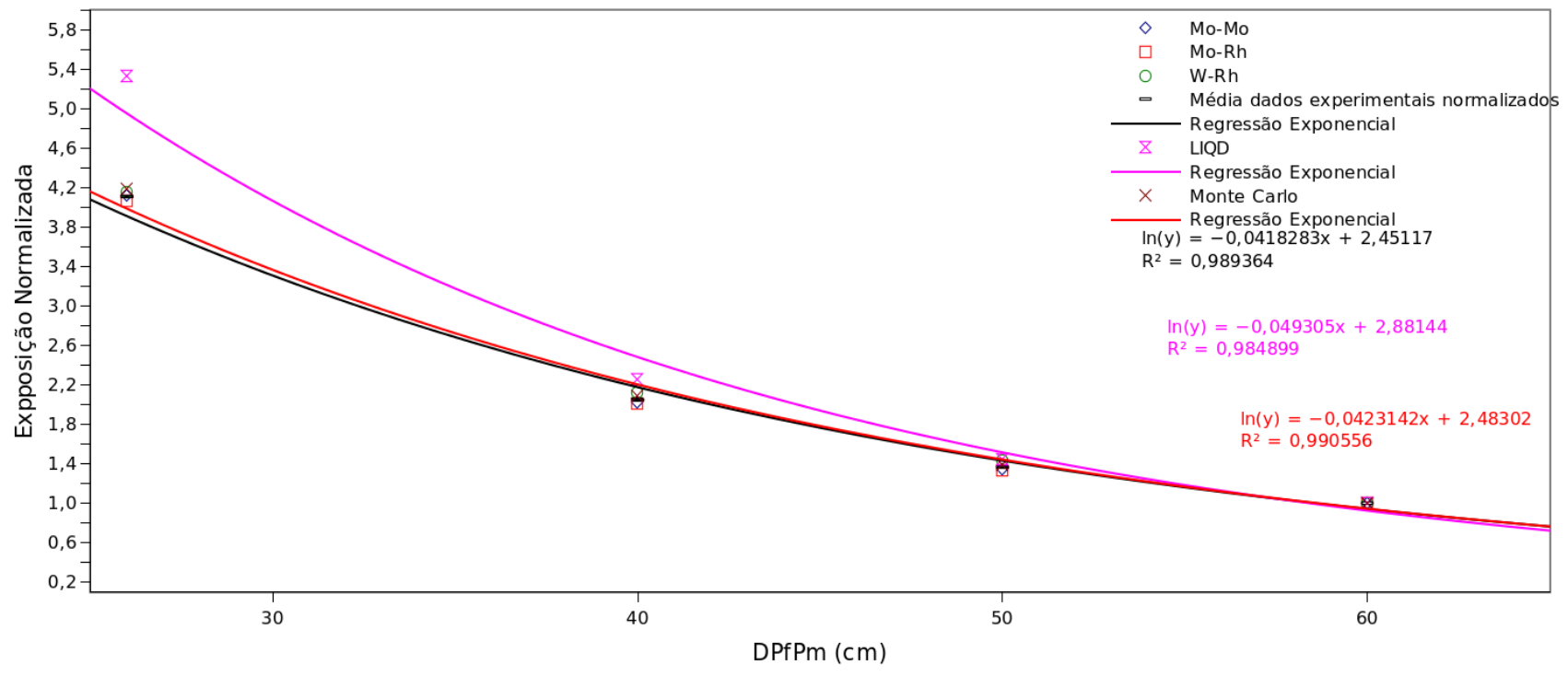


Figura 4. Gráfico que ilustra o comportamento das curvas das medidas de exposição normalizadas experimental, simulada no Geant4 e com aplicação do fator de correção do LIQD para as energias utilizadas em radiologia convencional considerando a medida/simulação a $40 \mathrm{~cm}$ como referência.

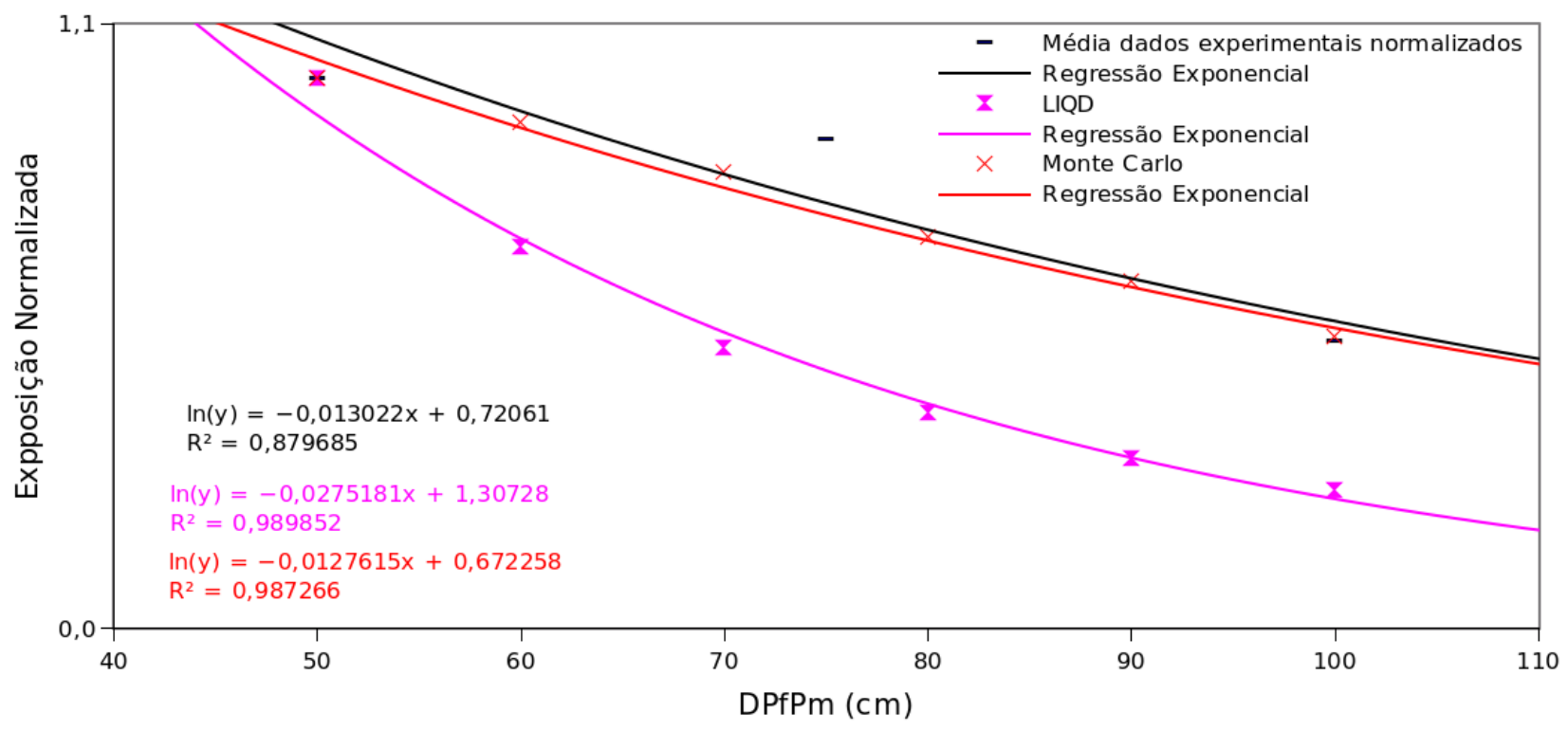

Como era de se esperar, as maiores diferenças foram determinadas para os pontos que ficaram mais distantes do ponto de referência. Para a mamografia as diferenças máximas determinadas entre as exposições normalizadas experimentais e as simuladas não ultrapassaram 4\%. Já as diferenças entre a LIQD para mamografia para as distâncias máximas de $34 \mathrm{~cm}$, entre os pontos $60 \mathrm{~cm}$ e $26 \mathrm{~cm}$, foram de 77,8\%. Para a área da radiologia convencional as diferenças calculadas entre exposições normalizadas experimentais e as simuladas, assim como nas simulações de mamografia não ultrapassaram 4\%. Já as diferenças entre a LIQD para radiologia convencional para as distâncias máximas de $50 \mathrm{~cm}$, entre os pontos $50 \mathrm{~cm}$ e $100 \mathrm{~cm}$, foram de 48\%. Conforme o esperado, feixes policromáticos menos energéticos e, consequentemente, com menor poder de penetração apresentaram maiores diferenças entre o fator de correção da LIQD e o valor de exposição normalizada. 
Analisando as figuras 2 e 3, percebe-se que as curvas experimental e simulada tem um comportamento menos acentuado do que aquela gerada pela LIQD. Isso se deve, provavelmente, pelo fato da simulação assim como a medida experimental considerarem o espalhamento da radiação pelo ar e pelos objetos da sala, como bucky e mesa. Como o fator de atenuação do ar é baixo (cita-se, como exemplo o valor deste coeficiente para $30 \mathrm{keV}$ como sendo $0,00043 \mathrm{~cm}^{-1}$ ) conforme dados do NIST (2014) o espalhamento pode acabar preponderando sobre a atenuação, o que justifica a suavidade da curva experimental.

Conforme o esperado, feixes policromáticos menos energéticos e, consequentemente, com menor poder de penetração, apresentaram maiores diferenças entre o fator de correção da LIQD e o valor de exposição experimental normalizado.

\section{CONCLUSÕES}

Os resultados mostram que a aplicabilidade de fatores de correção considerando somente a LIQD para espectros policromáticos aplicados em mamografia e radiologia convencional tem limitações significativa, sendo maiores as diferenças encontradas nos espectros aplicados a área da mamografia. As curvas de ajuste, apresentadas nas Figuras 2, 3 e 4, podem servir de base comparativa para avaliação de aplicabilidade da LIQD, dependendo da exatidão necessária para cada caso de interesse. Pode-se observar que, para cada todos os casos avaliados, quanto menor for a distância entre o ponto de referência e o ponto de interesse, menor será o erro sistemático aplicado ao caso de interesse.

\section{AGRADECIMENTO}

Os autores agradecem ao Hospital de Clínicas de Porto Alegre, especialmente aos Físicos Alexandre Bacelar e Rochelle Lykawka que possibilitaram a realização da coleta de dados experimental. Agradecemos também à Dra. Elaine Evani Streck pelas contribuições e sugestões singificativas técnicas e teóricas. 


\section{REFERÊNCIAS}

AAPM - American Association of Physicists in Medicine. Performance Specifications and Acceptance Testing for X-ray Generators and Automatic Exposure Control Devices. AAPM Report 14. AIP, 1:96, 1985.

AAPM - American Association of Physicists in Medicine. Protocols for the Radiation Safety Surveys of Diagnostic Radiological Equipment. AAPM Report 25. AAPM, 1989.

AAPM - American Association of Physicists in Medicine. Equipment Requirements and Quality Control for Mammography. AAPM Report 29. AAPM, 1990.

AGOSTINELLI, S. et al. GEANT 4 - a simulation toolkit. Nuclear Instruments and Methods in Physics Research Section A: Accelerators, Spectrometers, Detectors and Associated Equipment, v 506, Issue 3, p.250-303. 2003.

ALLISON, J. et al. Geant4 Developments and Applications. IEEE Transactions on Nuclear Science, v. 53, n. 1. 2006.

ATTIX, F. H. Gamma- and X-ray interactions in matter. In: Attix, F. H. Introduction to radiological physicists and radiation dosimetry. 2.ed, John Wiley \& Sons inc. p 124-159. 1986.

AUS, R.J.et al. A. Dependence of scatter on atomic number for $\mathrm{x}$ rays from tungsten and molybdenum anodes in the mammographic energy range.MedPhys, v.26, p.1306-1311. 1999.

ANVISA - Agência nacional de Vigilância Sanitária - Ministério da Saúde - Secretaria de Vigilância Sanitária. Diretrizes de Proteção Radiológica em Radiodiagnóstico Médica e Odontológic. ANVISA Portaria 453. p. 38. 1998.

CRANLEY K. et al. Catalogue of Diagnostic X-ray Spectra and Other Data. Report 78. The Institute of Physics and Engineering in Medicine. 1997.

DANCE, D.R. et al. Beast dosimetry using high-resolution voxel phantoms. Radiation Protection Dosimetry, v. 14, p. 359-363. 2005.

EUREF - European Reference Organisation for Quality Assured Breast Cancer Screening and Diagnostic Services. European protocol on dosimetry in mammography. EUR Report 16263. EN, Luxemburgo, p. 76. 1996. 
GINGOLD, E. L.; WU, X.; BARNES, G. T. Contrast and dose with Mo-Mo, Mo-Rh, and Rh-Rh target-filter combinations in mammography. Radiology, v.195, p. 639-644. 1995.

HOFF, G. Cálculo da Dose em Glândula Mamária, Utilizando o Código de Transporte de Monte Carlo MCNP, para as Energias Utilizadas em Mamografia. 2005. Tese (Doutorado em Biociências Nucleares) - Universidade do Estado do Rio de Janeiro, Rio de Janeiro. 2005.

HOFF, G. Efeito da filtração adicional de ródio e de molibdênio no contraste da imagem e dose glandular em mamografia. 2000. Dissertação (Mestrado em Biociências Nucleares) Universidade do Estado do Rio de Janeiro, Rio de Janeiro. 2000.

ICRU - International Commission on Radiation Units and Measurements. Tissue substitutes in Radiation Dosimetry and Measurement. ICRU Report 44, Maryland: ICRU. p. 189. 1989.

KRAMER, R. et al. All about FAX: a Female Adult voXel phantom for Monte Carlo calculation in radiation protection dosimetry. Phys. Med. Biol, v. 49, p. 5203-5216. 2004.

NG, K.P.; TANG, F. H. Monte Carlo simulation of x ray spectra in mammography. Phys. Med. Biol, v. 45, p. 1309-1318. 2000.

NIST - National Institute of Standard and Technology - Physical Reference Data. Available at: <http://physics.nist.gov/PhysRefData/contents.html /> Last accessed: 20. Aug, 2014.

TAKAHASHI, M.; KINASE, S.; R. KRAMER. Evaluation of counting efficiencies of awholebody counter using monte carlo simulation with voxel phantoms. Radiation Protection Dosimetry advance access, v. 144, p. 1-4. 2011.

WU, X. et al. Normalized average glandular dose in molybdenum target-rhodium filter and Rhodium target-Rhodium filter mammography. Med. Phys., v.193, n.3, p. 83-89. 1994.

WU, X.; BARNES, G. T.; TUCKER, D. M. Spectral dependence of glandular tissue dose in screen-film mammography. Radiology, v. 179, p. 143-148. 1991.

ZANKL, M. et al. Organ dose conversion coefficients for external photon irradiation of male and female voxel models. Phys. Med. Biol, v. 47, p. 2367-2385. 2002. 